\title{
Malformación de Chiari tipo I: evolución postoperatoria a dos años. Análisis de
}

\section{0 casos}

\author{
L. Alvarez-Betancourt; J. A. García-Rentaría; S.J. López-Ortega y A. Caldera-Duarte
}

Departamento de Neurocirugía. Hospital de Especialidades Centro Médico Nacional La Raza IMSS. México.

\section{Resumen}

Objetivo. Describir la evolución de 10 pacientes con Malformación de Chiari tipo I (MCI), desde el punto de vista clínico y de imagen mediante resonancia magnética (RM).

Método. A 10 pacientes con Malformación de Chiari se les realizó craniectomía suboccipital media, resección de arco posterior de $\mathrm{C} 1$ y plastia dural. A todos se les hizo un seguimiento clínico de dos años, y se hizo evaluación mediante RM a los seis meses del postoperatorio. Se correlaciona el tiempo de evolución preoperatoria, hallazgos de imagen mediante RM y la evolución clínica postoperatoria.

Resultados. La evolución clínica postoperatoria mostró una mejoría importante en cuanto a función sensitiva, correlacionándose con la involución de la siringohidromielia que acompañaba a esos casos, mejoría parcial de sus funciones cerebelosas y, en menor grado, mejoría de la alteración motora. Las manifestaciones de atrofia muscular no tuvieron modificaciones en el seguimiento a dos años.

Conclusiones. La MCI es una enfermedad congénita con múltiples manifestaciones clínicas que se van agravando conforme pasa el tiempo. Un diagnóstico oportuno y un tratamiento adecuado son indispensables para obtener los mejores resultado posibles desde el punto de vista neurológico.

PALABRAS CLAVE: Malformación de Chiari 1. Craniectomía suboccipital.

Chiari I malformation: postsurgical evolution after $\mathbf{2}$ years. Report of ten cases

\section{Summary}

Objective To describe the clinical and radiological evolution of ten patients with Chiari I malformation

Recibido: 6-11-03. Aceptado: 5-07-04
(CIM), treated surgically.

Method. Ten patients with Chiari I malformation underwent suboccipital craniectomy, laminectomy of $\mathrm{C} 1$ and dural patch grafting. All of them were followed for at least two years. A magnetic resonance imaging was performed 6 months after surgery.

Clinical preoperative evolution and time of installation, results of MRI and clinical postoperative evolution were correlated.

Results. In postoperative clinical evolution, we noted an important improvement in sensitive function, decrease of the syringohydromielia and partial improvement of cerebellar functions. Improvement of the motor alteration was less marked. During the two years after treatment the muscular atrophy did not change.

Conclusions. Chiari I malformation is a congenital disease with many clinical manifestations which usually increase with time. A suitable diagnostic studies as well as an appropriate treatment are needed to improve the neurological results.

KEY WORDS: Chiari I malformation. Suboccipital craniectom.

Introducción

Existen varias clasificaciones para determinar, según cada autor, el mejor abordaje posible y manejar así de manera integral las manifestaciones neurológicas que acompañan a la MCI. Así, Pillay y cols, la subdivide en: tipo A) cuando se acompaña de siringomielia y tipo B) sin ella ${ }^{12}$. Nagib la subdivide en IA) para pacientes con MCI, IB) para pacientes con MCI y siringomielia y IC) para pacientes con MCI, hidrocefalia, con o sin siringomielia ${ }^{6}$. Bindal y cols, por ejemplo, la subdividen en A) si los pacientes están asintomáticos, B) si los pacientes refieren signos o síntomas de compresión de tallo cerebral y S) si los pacientes refieren signos y síntomas secundarios a siringomielia ${ }^{2}$.

Clásicamente, se ha descrito la malformación de Chiari

Abreviaturas. IRM: imagen resonancia magnética. MCI: malformación Chiari tipo I. 
Tipo I (MCI) como el descenso anormal de las amígdalas cerebelosas de, al menos, $5 \mathrm{~mm}$ por debajo del foramen magno y por la cavitación secundaria de la médula espinal, hasta en un $88 \%$ de los $\operatorname{casos}^{3,8-10}$. Mediante cine-resonancia magnética se identificó la MCI como herniación amigdalina de menos de $5 \mathrm{~mm}$ si ésta se asocia a datos clínicos de compresión de tallo cerebral ${ }^{1,11}$. Para Williams, es de gran importancia la diferencia de presiones entre los compartimentos intracraneal e intraespinal. Con las maniobras de Valsalva,la presión intraespinal se eleva, a consecuencia del ingurgitamiento de las venas epidurales, el espacio subaracnoideo se comprime y la onda de presión se mueve en dirección cefálica hacia el espacio intracraneal; cuando la presión venosa espinal se disipa, se produce un flujo inverso de presión hacia el espacio intraespinal. Si este equilibrio se altera, por adherencias a nivel del foramen magno, se crea una presión diferencial entre ambos compartimentos y una ruta alternativa es el óbex que permanece vigente; otra es el desplazamiento caudal y progresivo de las amígdalas cerebelosas ${ }^{1,10,15}$. Para Nishikawa y cols, Ia patogénesis de la MCI se debe, posiblemente, a un defecto de desarrollo del encondrio occipital originado del mesodermo paraxial, con un aumento de la presión en la fosa craneal posterior que contiene las estructuras del tallo cerebral desarrolladas normalmente; a consecuencia de esto, se induce una herniación de las amígdalas cerebelosas así como un desplazamiento superior del tentorio ${ }^{7,14}$. En un estudio "post mortem", se encontró gliosis en el parénquima "perisirinx" y en los tractos de sustancia blanca dorsales adyacentes y en estos mismos, por arriba de los núcleos cuneatus y gracilis. Esto se explica como una degeneración walleriana de los tractos largos que dan lugar a áreas de alta intensidad en la resonancia magnética (RM), aún rostrales al extremo superior del "sirinx". Lo anterior explica la evolución tórpida en algunos casos. También se encontraron anormalidades en la microvasculatura que rodea al "sirinx", caracterizadas por la hipertrofia de la adventicia, ocasionando agrandamiento del espacio perivascular y permitiendo el paso del líquido cefalorraquídeo (LCR) del espacio subaracnoideo al parénquima medular que podría estar relacionado con la formación y progresión del "sirinx" $3,4,13$.

\section{Objetivo}

El objetivo de este estudio fue describir la evolución de 10 pacientes con MCI desde el punto de vista clínico y de imagen mediante RM, tratados quirúrgicamente con craniectomía suboccipital media, resección de arco posterior de $\mathrm{C} 1$ y plastia dural.

\section{Material y métodos}

De septiembre de 1998 a octubre del 2000, en el Ser- vicio de Neurocirugía del Hospital de Especialidades del Centro Médico Nacional Raza, se estudiaron 10 casos con diagnóstico de malformación de Chiari Tipo I. Todos reunían los siguientes criterios de inclusión: mayores de 17 años, que contaran con RM preoperatoria y de control a los seis meses, así como examen neurológico detallado preoperatorio y postoperatorio de control.

Del examen neurológico se analizaron los siguientes parámetros: 1. Manifestaciones medulares: alteraciones sensitivas, alteraciones motoras, trastornos en el control de esfínteres. 2. Manifestaciones cerebelosas: nistagmo, ataxia, disartria, Romberg. 3. Manifestaciones de tallo cerebral: sordera, disfagia, disfonía, disestesia trigeminal.

A todos los pacientes se les realizó descompresión de la unión craneovertebral consistente en craniectomía suboccipital media a $3 \mathrm{~cm}$ de la línea media por debajo de la protuberancia occipital externa, resección del arco posterior de $\mathrm{Cl}$, liberación de adherencias aracnoideas, si estaban presentes, y plastia dural en forma de "Y" con injerto autólogo de aponeurosis local.

\section{Resultados}

Se analizaron 10 casos de MCI; 6 hombres y 4 mujeres con edades de 22 a 60 años (promedio 35). Ninguno contaba con antecedentes familiares de MCI o relacionados. El tiempo de evolución del padecimiento, hasta el momento de la cirugía, varió de 1 a 15 años (promedio 5.5 años).

El período de seguimiento fue de 24 a 32 meses (promedio 25.7). Respecto al estado laboral de los pacientes, 6 no trabajaban previamente por causas distintas a la enfermedad que nos ocupa; los otros 4 sí trabajaban previamente $\mathrm{y}$ estuvieron en condiciones de reincorporarse a su trabajo después de la operación (casos 2,3,4 y 6); uno de ellos (caso 6) tuvo que ser reubicado en su centro de trabajo. Tabla 1.

\section{Características clínicas}

Manifestaciones de tallo cerebral: En esta parte se exploró sordera (caso 9), disfagia (casos 5 y 7), disfonía (casos 1, 5 y 7), disestesia trigeminal (caso 5) vértigo (casos 5 y 8). La evolución postoperatoria fue hacia la desaparición de todas las manifestaciones, con excepción de la sordera (caso 9), que estaba en relación a un clima laboral adverso.

Manifestaciones medulares: Se encontraron alteraciones sensitivas caracterizadas por hipoestesia (casos 2, 3 y 4), parestesias (0 casos), y disociación termoalgésica (casos 1, 5, 6, 8, 9 y 10). En todos los casos hubo resolución de estas alteraciones sensitivas en el período que varió desde la primera semana postoperatoria al tercer mes postoperatorio. Alteraciones motoras: se encontraron alte- 
Tabla 1

Parámetros clínicos

\begin{tabular}{|l|c|c|c|c|c|c|c|c|c|c|}
\hline Paciente & $\mathbf{1}$ & $\mathbf{2}$ & $\mathbf{3}$ & $\mathbf{4}$ & $\mathbf{5}$ & $\mathbf{6}$ & $\mathbf{7}$ & $\mathbf{8}$ & $\mathbf{9}$ & $\mathbf{1 0}$ \\
\hline Edad & 32 & 25 & 23 & 42 & 22 & 33 & 58 & 29 & 60 & 26 \\
Sexo & $\mathrm{F}$ & $\mathrm{M}$ & $\mathrm{M}$ & $\mathrm{M}$ & $\mathrm{F}$ & $\mathrm{M}$ & $\mathrm{F}$ & $\mathrm{F}$ & $\mathrm{M}$ & $\mathrm{M}$ \\
\hline Antecedentes familiares & $\mathrm{No}$ & $\mathrm{No}$ & $\mathrm{No}$ & $\mathrm{No}$ & $\mathrm{No}$ & $\mathrm{No}$ & $\mathrm{No}$ & $\mathrm{No}$ & No & No \\
\hline Tiempo de evolución & $12 \mathrm{~A}$ & $1 \mathrm{~A}$ & $4 \mathrm{~A}$ & $3 \mathrm{~A}$ & $2 \mathrm{~A}$ & $5 \mathrm{~A}$ & $6 \mathrm{~A}$ & $2 \mathrm{~A}$ & $15 \mathrm{~A}$ & $5 \mathrm{~A}$ \\
\hline Tiempo de seguimiento & $26 \mathrm{M}$ & $32 \mathrm{M}$ & $27 \mathrm{M}$ & $27 \mathrm{M}$ & $24 \mathrm{M}$ & $24 \mathrm{M}$ & $25 \mathrm{M}$ & $24 \mathrm{M}$ & $24 \mathrm{M}$ & $24 \mathrm{M}$ \\
\hline Estado laboral - (Normal) & $\mathrm{No}$ & $\mathrm{Si}$ & $\mathrm{Si}$ & $\mathrm{Si}$ & $\mathrm{No}$ & $\mathrm{Si}$ & No & No & No & No \\
\hline
\end{tabular}

F: Femenino. M: Masculino. A: Años. M: Meses. Estado laboral: Si trabajaban antes de la cirugía, No trabajaban antes de la cirugía.

Tabla 2

Manifestaciones clínicas

\begin{tabular}{|c|c|c|c|l|l|}
\hline Número de caso & Nistagmo & Ataxia & Romberg & Alt. sensit. & Alt. motora \\
\hline $\mathbf{1}$ preqx & No & Izquierda & No & Nivel C3 & Tetrap. $3 / 5$ \\
postqx & No & No & No & Normal & Tetrap. $-4 / 5$ \\
\hline $\mathbf{2}$ preqx & No & No & No & C2-C8 der & $-4 / 5$ mtd \\
postqx & No & No & No & Normal & Igual \\
\hline 3 preqx & Grado III & Indistinta & Si & C6-C8 izq & Normal \\
postqx & Grado I & No & No & Normal & Normal \\
\hline $\mathbf{4}$ preqx & No & No & No & C6-C7 izq & $4 / 5$ Ms Ts \\
postqx & No & No & No & Normal & Normal \\
\hline $\mathbf{5}$ preqx & Grado I & Si & Si & Hipoest.bilateral & Normal \\
postqx & Grado I & Si & Si & Normal & Normal \\
\hline $\mathbf{6}$ preqx & No & No & No & DTA C3-L1 & C6-C8 -4/5 \\
postqx & No & No & No & Mejoró & Igual \\
\hline 7 preqx & Grado II & Derecha & Si & Normal & Normal \\
postqx & No & No & No & Normal & Normal \\
\hline $\mathbf{8}$ preqx & Grado II & Indistinta & Si & DTA mtd & Normal \\
postqx & Grado I & No & No & Normal & Normal \\
\hline $\mathbf{9}$ preqx & Grado I & No & No & L1 der T12 izq & Mpi1/5 mpd 3/5 \\
postqx & Grado I & No & No & Normal & Igual \\
\hline $\mathbf{1 0}$ preqx & No & No & No & DTA mtd & C6-C8 -4/5 \\
postqx & No & No & No & Normal & Igual \\
\hline
\end{tabular}

Alt. Sensit: Alteración sensitiva. Alt: Alteración. Tretap.: tetraparesia. Der: derecha. Izq: Izquierda. Mpd: miembro pélvico derecho. Mtd: miembro torácico derecho. Mpi: miembros pélvico izquierdo. MsTs: miembros torácicos. DTA: Disociación termoalgésica.

raciones de la fuerza (casos $1,2,4,6,9,10)$ Babinski (casos $1,2,4,5,6,8,9,10)$ y la presencia de atrofia de músculos interóseos (casos 1, 6, 9, 10). La evolución postoperatoria de la fuerza fue mala; sólo hubo una leve mejoría de un paciente (caso 4); el resto de los pacientes se mantuvieron sin cambios durante el período de seguimiento; respecto a la atrofia de músculos interóseos y al signo de Babinski, no se observó mejoría. Tabla 2. 

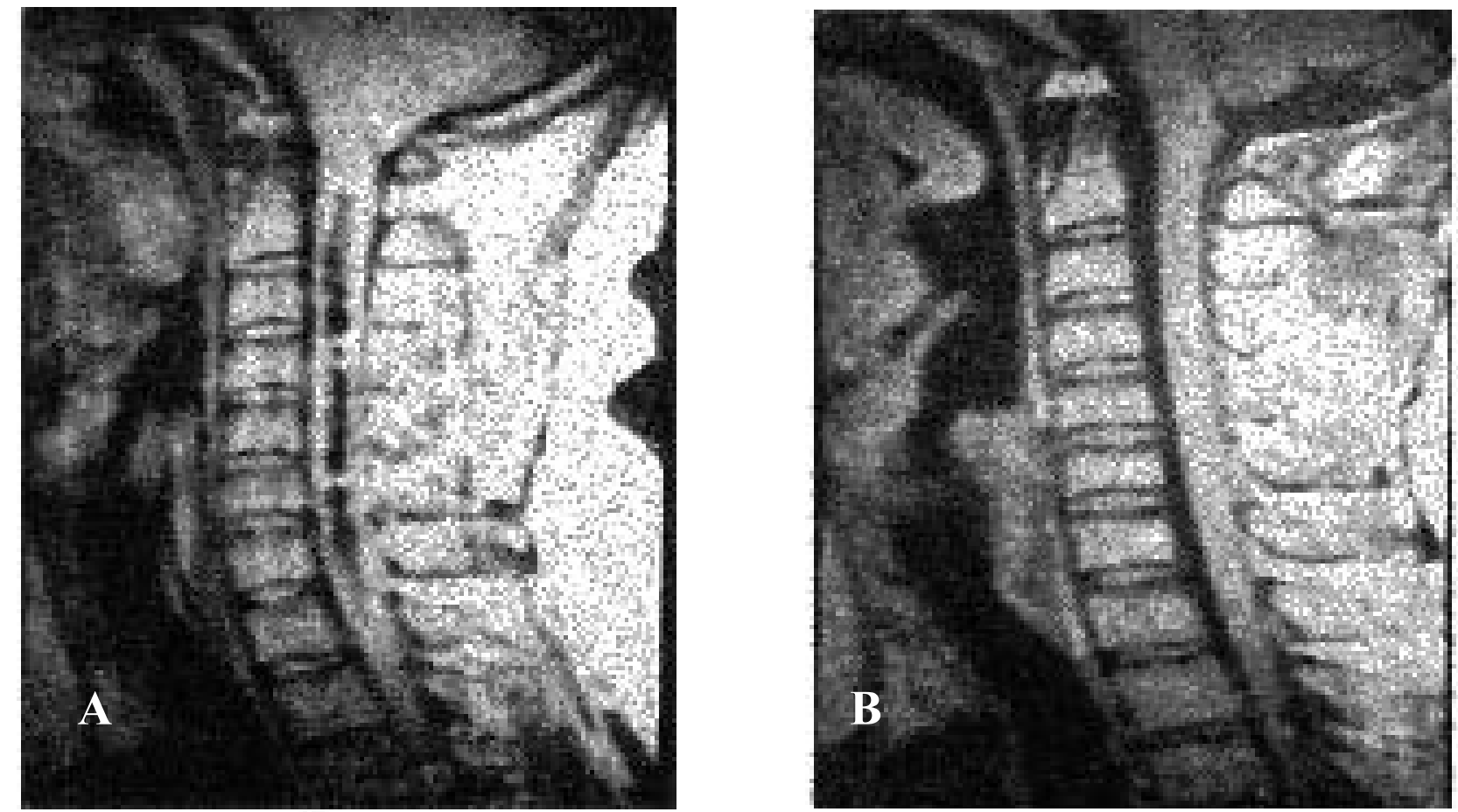

Figura 1 A: Imagen de RM de columna cervical preoperatorio donde se nota la marcada siringohidromielia cervical con descenso de la amígdalas cerebelosas. 1 B imagen de RM 6 meses después del tratamiento quirúrgico, craniectomía suboccipital media, resección de arco posterior de C1 y plastía dural, observándose desaparición de la siringohidromielia y ascenso de las amígdalas cerebelosas. (Caso 3).

Manifestaciones cerebelosas: Se encontró nistagmo en 5 pacientes ( $\operatorname{casos} 3,5,7,8$, y 9), ataxia en 5 pacientes (casos $1,3,5,7$ y 8 ), dismetría 3 pacientes (casos 5,7 y 8 ) disartria 2 pacientes (casos 5 y 8 ) signo de Romberg en 4 pacientes (casos $3,5,7$ y 8 ).

La evolución postoperatoria fue hacia la mejoría, encontrándose disminución o desaparición del nistagmo en 3 pacientes (persistiendo en los casos 5 y 9); respecto a la ataxia desapareció en 4 casos, persistiendo en el caso 5; en la dismetría hubo desaparición de ésta en dos casos (persistiendo en el caso 5). En los dos casos con disartria hubo desaparición gradual de ésta hasta el lenguaje normal. El signo de Romberg desapareció en 3 casos pero persistió en el caso 5. Tabla 2.

\section{Hallazgos en RM}

A todos los pacientes se les realizó RM preoperatoria, con la cual se confirmó el diagnóstico; 6 meses después de la cirugía se hizo el mismo estudio. Por los hallazgos en la RM preoperatoria los agrupamos en: Grupo I: 8 pacientes con siringohidromielia cervical; Grupo II: 1 paciente con siringohidromielia cervicotorácica y Grupo III: 1 paciente sin siringohidromielia.

Se encontró en la RM postoperatoria que de los pacien- tes que pertenecían al grupo I, en 5 disminuyó considerablemente su diámetro y longitud (casos 1, 2, 4, 6, 8); en 2 desapareció completamente (casos 3 y 5) (Fig. 1) y en uno permaneció sin cambios (caso 10).

El paciente del grupo II, (caso 9) permaneció sin cambios. El paciente del grupo III, (caso 7) no tenía siringohidromielia. En todos los casos operados observamos ascenso y redondeamiento de las amígdalas, que previo a la operación se mostraban, además de anormalmente descendidas, en ángulo agudo.

Encontramos una serie de alteraciones óseas asociadas a la MCI, caracterizadas por escoliosis cervicotorácica (casos 1 y 2) arco posterior de $\mathrm{C} 1$ hipoplásico y fusionado a C2 (casos 4, 5 y 7) y en un paciente (caso 6) se evidenció fractura neuropática de la cabeza humeral izquierda.

Se diagnosticó hidrocefalia asociada, con hipertensión intracraneal en 1 paciente (caso 8) que necesitó la colocación de una derivación ventrículo peritoneal para su tratamiento.

\section{Discusión}

La MCI es una patología congénita que se manifiesta desde la infancia hasta la edad adulta. Las características clínicas son muy variadas y están en relación directa con la 
fisiopatología de ésta enfermedad ${ }^{3,5}$. Hemos observado que a mayor período de evolución, antes del diagnóstico de la enfermedad, el cuadro clínico es más evidente. En nuestra serie de casos encontramos una remisión completa de las manifestaciones medulares del tipo sensitivo; esta mejoría se mantuvo durante todo el período de seguimiento. Así mismo, las manifestaciones cerebelosas y del tallo cerebral remitieron gradualmente con el paso del tiempo en los primeros 3 meses de seguimiento. Hubo un paciente (caso 8), que en el postoperatorio inmediato presentó incapacidad para deglutir, ausencia del reflejo nauseoso, trastornos en el patrón respiratorio y exacerbación del nistagmo. Fue tratado de manera conservadora con recuperación completa de estos trastornos al tercer día postoperatorio.

En todos los casos observamos que, una vez presentada la mejoría en alguna manifestación, ésta no empeoraba con el paso del tiempo, con excepción de 1 paciente (caso 10) que, al cabo de 24 meses de seguimiento, nuevamente presentó disociación termoalgésica y la RM de control puso de manifiesto incremento en el diámetro y longitud del sirinx. La evolución y los hallazgos clínicos apoyan la teoría de la obstrucción del libre flujo de líquido cefalorraquídeo a nivel de la unión craneovertebral, con descenso de líquido durante la sístole y ascenso durante la diástole. Al verse modificado este mecanismo se inicia el fenómeno compresivo sobre el espacio subaracnoideo medular y la formación gradual y progresiva del sirinx, dando como resultado un síndrome medular central progresivo ${ }^{10}$. No encontramos una relación directa entre el tamaño del "sirinx", y las manifestaciones medulares, pero sí una regresión del sirinx en el postoperatorio, corroborado por RM. El fenómeno compresivo a nivel del cerebelo y del tallo cerebral parece estar en relación con una fosa posterior pequeña por subdesarrollo óseo a este nivel ${ }^{7}$.

Aquellas manifestaciones clínicas que no desaparecieron en el postoperatorio ni en el período de seguimiento podrían estar relacionadas con el daño permanente de las vías nerviosas o de sus núcleos.

Nosotros observamos cierta relación entre el tiempo de evolución de la enfermedad y la disminución o desaparición del sirinx, ya que, promediando el tiempo de evolución en cada uno de los grupos de pacientes, encontramos que en el grupo I hubo un período de evolución promedio de 4.2 años, en el grupo II fue de 15 años y en el grupo III fue de 6 años.

\section{Bibliografía}

1. Bhadelia, R., Bodgan, A., Wolpert, S., Lev, S, Appigmani, B., Heilman, C.: Cerebrospinal fluid flow wave forms: analysis in patients with Chiari 1 malformation by means of gated phase-contrast MR imaging velocity meassurements. Radiology. 1996: 19: 195-202.
2. Bindal, A., Dunsker, S., Tew, J.: Chiari I malformation: classification and management. Neurosurgery. 1995: 37: 1069-1074.

3. Dyste, G., Menezes, A., Van Gilder, J.: Synptomatic Chiari malformations. An analysis of presentation, management and long-term outcome. J Neurosurg. 1989: 71: 159-168

4. Grabb, P., Mapstone, T., Oakes, J.: Ventral brain stem compression in pediatric and young adults patients with Chiari I malformations. Neurosurgery. 1999: 44: 520-527.

5. Milhorat, T., Chou, M.: Chiari I malformation redefined: clinical and radiographic findings for 364 symptomatic patients. Neurosurgery. 1999: 44: 1005-1017.

6. Nagib, M.: An approach to symptomatic children (ages 4 -14) with Chiari type I malformation. Pediatr Neurosurg. 1994: 5: 21-31.

7. Nishikawa, M., Sakamoto, H., Hakuba, A.: Patogenesis of Chiari malformation: a morphometric study of the posterior cranial fosa. J Neurosurg. 1997: 86: 40-44.

8. Nogués, M., Gené, R., Benarroch, E., Leiguarda, R., Calderón, C.: Respiratory disturbances during sleep in syringomyelia and syringobulbia. Neurology. 1999: 52: 177-198.

9. Oakes, W.: Chiari malformations, hydromielia, syringomielia in Rengashary. Neurosurgery. 1995: 3: 593-616.

10. Oldfteld, E., Muraszko, K.: Pathophysiology of syringomielia associated with Chiari I malformation of the cerebelar tonsils. J Neurosurg. 1994: 80: 3-15.

11. Pujol, J., Roing, C., Capdevila, A., Pou, A., et al,: Motion of the cerebellar tonsils in Chiari type I malformation studies by cine phase-constrast MRI. Neurology. 1995: 45: 1746-1753.

12. Pillay, P.K., Awad, I.A., Little, J.R., Hahn, J.F.: Symptomatic Chiari malformation in adults: A new classification based on magnetic resonance imaging with clinical and prognostic significance. Neurosurgery. 1991: 28: 639-645.

13: Rhoton, A.: Microsurgery of syringomielia and syringomielic cord symdron. En Schmidek, H.H. and Sweet, W.H., ed. W.B. Saunders Co. Philadelphia 1995. 2: 1745-1764.

14. Vanaclocha, V., Sais-Sapena, N., García, C.: Surgical technique for Iranio-cervical decompression in syringomyelia associated with Chiari I Malformation. Acta Neurochir (Wien). 1997: 139: 529-540.

15. Williams, B.: The distending force in the production of syringomyelia. Lancet. 1969: 2: 189-193

Alvarez-Betancourt, L.; García-Rentaría, J.A.; LópezOrtega, S.J.; Caldera-Duarte, A.: Malformación de Chiari tipo I: evolución postoperatoria a dos años. Análisis de 10 casos. Neurocirugía 2005; 16: 34-38.

Correspondencia postal: Leonardo Alvarez Betancourt. Departamento de Neurocirugía del Hospital de Especialidades CMN La Raza. Seris y Zaachila S/N Col. La Raza, $6^{\circ}$ piso, Deleg. Azcapotzalco C.P. 02990 México DF. E-mail: azbtlo@hotmail.com 